ARTICLE

Check for updates

https://doi.org/10.1038/s41467-020-17999-4

\title{
Controlling spin current polarization through non-collinear antiferromagnetism
}

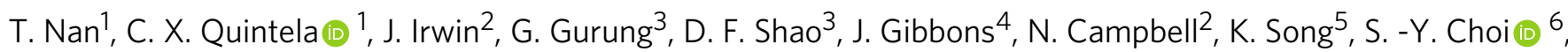
L. Guo', R. D. Johnson 7,8,9 , P. Manuel (1) 9 , R. V. Chopdekar (10 10, I. Hallsteinsen ${ }^{10,11}$, T. Tybell (1) 11, P. J. Ryan ${ }^{12,13}$, J. -W. Kim², Y. Choi (iD ${ }^{12}$, P. G. Radaelli ${ }^{7}$, D. C. Ralph (i) 4,14, E. Y. Tsymbal (i) ${ }^{3}$, M. S. Rzchowski ${ }^{2} \&$

C. B. Eom (10 ${ }^{1 \mathrm{~W}}$

The interconversion of charge and spin currents via spin-Hall effect is essential for spintronics. Energy-efficient and deterministic switching of magnetization can be achieved when spin polarizations of these spin currents are collinear with the magnetization. However, symmetry conditions generally restrict spin polarizations to be orthogonal to both the charge and spin flows. Spin polarizations can deviate from such direction in nonmagnetic materials only when the crystalline symmetry is reduced. Here, we show control of the spin polarization direction by using a non-collinear antiferromagnet $\mathrm{Mn}_{3} \mathrm{GaN}$, in which the triangular spin structure creates a low magnetic symmetry while maintaining a high crystalline symmetry. We demonstrate that epitaxial $\mathrm{Mn}_{3} \mathrm{GaN}$ /permalloy heterostructures can generate unconventional spin-orbit torques at room temperature corresponding to out-of-plane and Dresselhaus-like spin polarizations which are forbidden in any sample with two-fold rotational symmetry. Our results demonstrate an approach based on spin-structure design for controlling spin-orbit torque, enabling high-efficient antiferromagnetic spintronics.

\footnotetext{
${ }^{1}$ Department of Materials Science and Engineering, University of Wisconsin-Madison, Madison, WI 53706, USA. ${ }^{2}$ Department of Physics, University of Wisconsin-Madison, Madison, WI 53706, USA. ${ }^{3}$ Department of Physics and Astronomy \& Nebraska Center for Materials and Nanoscience, University of Nebraska, Lincoln, NE 68588, USA. ${ }^{4}$ Laboratory of Atomic and Solid State Physics, Cornell University, Ithaca, NY 14853 , USA. ${ }^{5}$ Department of Materials Modeling and Characterization, KIMS, Changwon 51508, South Korea. ${ }^{6}$ Department of Materials Science and Engineering, POSTECH, Pohang 37673 , South Korea. ${ }^{7}$ Clarendon Laboratory, Department of Physics, University of Oxford, Parks Road, Oxford OX1 3PU, UK. ${ }^{8}$ ISIS Facility, Rutherford Appleton Laboratory, Chilton, Didcot OX11 0QX, UK. ${ }^{9}$ Department of Physics and Astronomy, University College London, Gower Street, London WC1E 6BT, UK. ${ }^{10}$ Advanced Light Source, Lawrence Berkeley National Laboratory (LBNL), 1 Cyclotron Road, Berkeley, CA 94720, USA. ${ }^{11}$ Department of Electronic Systems, Norwegian University of Science and Technology, Trondheim 7491, Norway. ${ }^{12}$ Advanced Photon Source, Argonne National Laboratory, Argonne, IL 60439, USA.

${ }^{13}$ School of Physical Sciences, Dublin City University, Dublin 11, Ireland. ${ }^{14}$ Kavli Institute at Cornell for Nanoscale Science, Ithaca, NY 14853, USA.

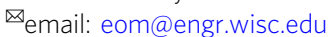


C urrent-induced spin-orbit torque enables highly efficient manipulation of magnetization for spintronic applications ${ }^{1-8}$. In the classical picture of current-induced magnetization dynamics ${ }^{9,10}$ (Fig. 1f), charge currents in a multilayer sample flowing along the in-plane direction ( $\mathbf{x}$ direction) can generate out-of-plane spin currents (flowing in the $\mathbf{z}$ direction) that have spin polarization $\boldsymbol{\sigma}$, required by symmetry to be along the $\mathbf{y}$ direction corresponding to a Rashba-like spin polarization. This particular spin current can give rise to an anti-damping spin torque in an adjacent ferromagnet, which has magnetization vector $\mathbf{m}$, of the form $\mathbf{m} \times(\mathbf{m} \times \mathbf{y})$. The anti-damping torque is responsible for efficient magnetization manipulation when the torque is collinear with the magnetization leading to a direct change of the effective magnetic damping. But as the antidamping torque is restricted to lie along an in-plane direction, it is efficient for manipulating only samples with magnetic anisotropy along the in-plane $\mathbf{y}$ axis, not along the out-of-plane (z) direction or collinear with the current (x direction). Such a limitation of controlling the spin-torque or spin polarizations makes the switching of magnetic devices with magnetic anisotropy along the $\mathbf{x}$ or $\mathbf{z}$ axis nondeterministic and much less efficient (than that along $\mathbf{y}$ direction $)^{11}$.

To efficiently and deterministically drive, for example, perpendicularly-magnetized devices that are preferred for highdensity memories, an out-of-plane anti-damping torque is required. Such an out-of-plane spin-torque can originate from spin-orbit scattering from ferromagnetic interfaces ${ }^{12-14}$, or can arise at the interface in systems with reduced symmetry, such as in bilayers of a non-magnetic transition-metal dichalcogenide and a ferromagnetic metal ${ }^{15}$. However, these effects based on interface or heterostructure engineering have not been demonstrated to be strong enough for practical anti-damping switching. Here we demonstrate an alternative strategy to achieve unconventional spin-orbit torques, based on long-range non-collinear magnetic order within the bulk of the spin-source layer. In particular, we use the spin-Hall effect in epitaxial thin films of $\mathrm{Mn}_{3} \mathrm{GaN}$, a metallic antiferromagnet that has a $120^{\circ}$ triangular spin texture, which reduces the symmetry sufficiently to allow spin current generation with different spin polarization directions to generate unconventional spin-torques. In heterostructures of epitaxial $\mathrm{Mn}_{3} \mathrm{GaN} /$ permalloy, we observe not only the out-of-plane antidamping torque, but also the anti-damping torque corresponding to a Dresselhaus-like spin polarization ${ }^{16-18}$, besides the conventional Rashba-like symmetry. When the non-collinear spin texture is eliminated by heating above the Néel temperature of $\mathrm{Mn}_{3} \mathrm{GaN}(345 \mathrm{~K})$, the unconventional spin-torques go to zero. Such a control of the spin polarizations is coincident with our symmetry analysis and theory calculation upon the magnetic space groups across the Néel transition. Although the spin-Hall effect has been previously demonstrated in antiferromagnetic thin films, only Rashba-like symmetry has been observed ${ }^{19-22}$.

$\mathrm{Mn}_{3} \mathrm{GaN}$ is a metallic nitride with the antiperovskite crystal structure $^{23,24}$ (identical to the perovskite structure, but with anion and cation positions interchanged) and a lattice parameter close to that of commonly used perovskite oxide substrates. In the bulk, it is known to exhibit antiferromagnetic ordering with a noncollinear $\Gamma^{5 \mathrm{~g}}$ Kagome-like structure (magnetic space group: $\mathrm{R} \overline{3} \mathrm{~m}$ ) stabilized by the magnetic frustration of the $\mathrm{Mn}$ atoms in the (111) plane (Fig. 1a) ${ }^{23,25}$. In the (001) plane of $\mathrm{Mn}_{3} \mathrm{GaN}$ (Fig. 1b), the (110) plane is the only mirror plane. In this low-symmetry a

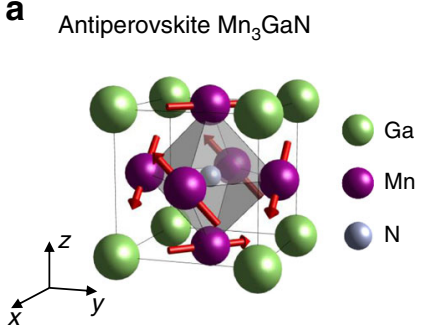

d

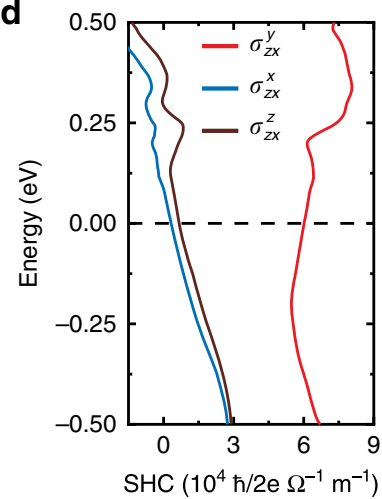

b

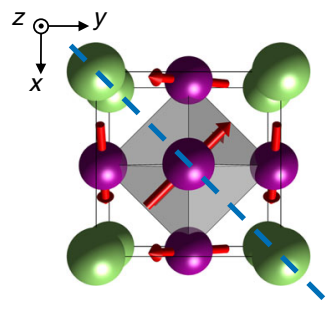

With non-collinear spins

e

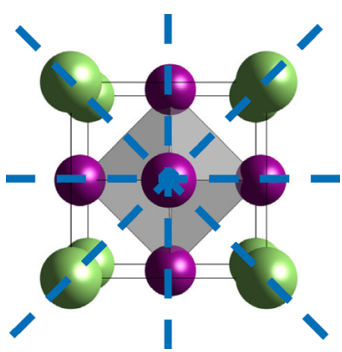

Without non-collinear spins
C

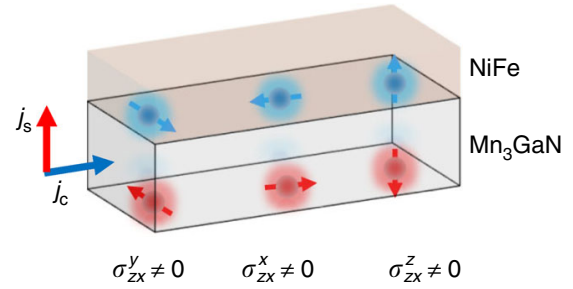

f

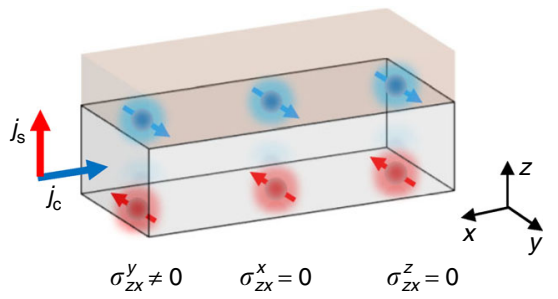

Fig. 1 The concept of the unconventional spin-Hall effect in $\mathbf{M n}_{\mathbf{3}} \mathbf{G a N}$. a The crystallographic unit cell of antiperovskite Mn ${ }_{3} \mathrm{GaN}$ with the antiferromagnetic $\Gamma^{5 \mathrm{~g}}$ spin structure where $\mathrm{Mn}$ spins (arrows) form a Kagome-type lattice in the (111) plane. $\mathbf{x}, \mathbf{y}$, and $\mathbf{z}$ correspond to the cubic [100], [010], and [001] axes, respectively. b Spin structure of $\mathrm{Mn}_{3} \mathrm{GaN}$ projected onto the (001) plane. The blue dashed line corresponds to the (110) mirror plane. c Schematic illustrations of the Py/Mn ${ }_{3} \mathrm{GaN}$ bilayer and the allowed spin-Hall spin polarization in the low-symmetry state (a). This indicates nonzero spin-Hall conductivities $\sigma_{z x^{\prime}}^{y} \sigma_{z x^{\prime}}^{x}$ and $\sigma_{z x^{\prime}}^{z}$ which correspond to spin polarizations along $\mathbf{y}, \mathbf{x}$, and $\mathbf{z}$ direction, respectively (with the charge current along $\mathbf{x}$ and spin flow along $\mathbf{z}$ ). d Calculated spin-Hall conductivities $\sigma_{z x}^{y}, \sigma_{z x^{\prime}}^{x}$ and $\sigma_{z x}^{z}$ for $\mathrm{Mn}_{3} \mathrm{GaN}$ in the antiferromagnetic phase as a function of Fermi energy. e Crystal structure of $\mathrm{Mn}_{3} \mathrm{GaN}$ without non-collinear spin structure (i.e., above the antiferromagnetic transition temperature $T_{\mathrm{N}}$ ) in the (001) plane, which gives rise to a high-symmetry state. $\mathbf{f}$ Allowed spin polarization in the high-symmetry state, where only the conventional spin-Hall conductivity $\sigma_{z x}^{y}$ is nonzero. 
state, we find that charge currents along $\mathbf{x}$ generate unconventional anti-damping torque components in the form of $\boldsymbol{\tau}_{x} \propto$ $\mathbf{m} \times(\mathbf{m} \times \mathbf{x})$ and $\boldsymbol{\tau}_{z} \propto \mathbf{m} \times(\mathbf{m} \times \mathbf{z})$ in addition to the conventional $\boldsymbol{\tau}_{y} \propto \mathbf{m} \times(\mathbf{m} \times \mathbf{y})$, which correspond to spin currents with $\boldsymbol{\sigma}$ along $\mathbf{x}, \mathbf{z}$, and $\mathbf{y}$, respectively (Fig. 1c). These spin currents have corresponding spin-Hall conductivities $\sigma_{z x}^{x}, \sigma_{z x}^{z}$, and $\sigma_{z x}^{y}$ (in the form of $\sigma_{j k}^{i}$, where $i, j$ and $k$ denote the spin polarization, spin flow and charge flow directions). The symmetry allowed, and experimentally observed, non-zero spin-Hall conductivities are consistent with our linear theory calculation (Supplementary Note 9). Figure $1 \mathrm{~d}$ shows that the $\sigma_{z x}^{x}, \sigma_{z x}^{z}$, and $\sigma_{z x}^{y}$ calculated by using the bulk $\mathrm{Mn}_{3} \mathrm{GaN}$ band structure are large within a wide energy window around the charge neutrality point, implying the existence of a sizable spin-Hall current even in the presence of charge carrier doping by defects. Above the antiferromagnetic-toparamagnetic transition temperature (Néel temperature $T_{\mathrm{N}}$ ), disordered spins give rise to a high-symmetry state (space group: $P m \overline{3} m$ ) having 4 mirror planes in the crystal lattice (Fig. 1e), and consequently only the conventional spin-Hall conductivity $\sigma_{z x}^{y}$ can be non-zero. We list the matrices of the spin-Hall conductivity tensors, obtained from symmetry analysis and calculations, for $\mathrm{Mn}_{3} \mathrm{GaN}$ in antiferromagnetic and paramagnetic phases in the Supplementary Table 1 .

\section{Results}

Epitaxial $\mathrm{Mn}_{3} \mathrm{GaN}$ thin films were grown on (001) $\left(\mathrm{La}_{0.3} \mathrm{Sr}_{0.7}\right)$ $\left(\mathrm{Al}_{0.65} \mathrm{Ta}_{0.35}\right) \mathrm{O}_{3}$ (LSAT) substrates by reactive magnetron sputtering with in-situ reflection high-energy electron diffraction (RHEED, see "Methods"). The out-of-plane X-ray diffraction around the (002) LSAT substrate peak shows an epitaxial $\mathrm{Mn}_{3} \mathrm{GaN}$ film (Fig. 2a). The distinct Kiessig fringes around the $\mathrm{Mn}_{3} \mathrm{GaN}$ (002) peak and the streaky RHEED pattern of the $\mathrm{Mn}_{3} \mathrm{GaN}$ film surface (Fig. 2a inset) indicate a high crystalline quality and a smooth film surface. We also confirmed the cubeon-cube epitaxial relationship between the $\mathrm{Mn}_{3} \mathrm{GaN}$ film and underlying LSAT substrate (Supplementary Note 1). Ferromagnetic permalloy $\mathrm{Ni}_{81} \mathrm{Fe}_{19}$ (Py) and $\mathrm{Cu}$ (as a spacer layer) thin films were then deposited in situ on $\mathrm{Mn}_{3} \mathrm{GaN}$ to form the $\mathrm{Py} / \mathrm{Cu} /$ $\mathrm{Mn}_{3} \mathrm{GaN}$ tri-layer, and finally were patterned into device bars for spin-torque measurements. In Fig. 2b, we show the crosssectional filtered STEM-HAADF image of a $30-\mathrm{nm} \mathrm{Mn}_{3} \mathrm{GaN}$ film on LSAT capped with 10-nm Py, which reveals sharp interfaces between both $\mathrm{Mn}_{3} \mathrm{GaN} / \mathrm{LSAT}$ (left) and $\mathrm{Py} / \mathrm{Mn}_{3} \mathrm{GaN}$ (right). Atomic force microscope images of the sample surface indicate an atomically-smooth surface with a surface roughness of $\sim 0.3 \mathrm{~nm}$.
Using neutron diffraction, we determined that our 250-nm $\mathrm{Mn}_{3} \mathrm{GaN}$ films order with the bulk antiferromagnetic triangular $\Gamma^{5 \mathrm{~g}}$ spin structure below a Néel temperature of $T_{\mathrm{N}}=\sim 350 \mathrm{~K}$ (Supplementary Note 3), consistent with that for the thinner 20$\mathrm{nm}$ films having $T_{\mathrm{N}}=345 \mathrm{~K}$, but higher than that for polycrystalline bulk samples $\left(T_{\mathrm{N}} \sim 290 \mathrm{~K}\right)$, possibly due to less grain boundaries and slight nitrogen deficiency in thin film samples (Supplementary Note 2). Using X-ray magnetic linear and circular dichroism with photoemission electron microscopy, we observe antiferromagnetic domains with size on the order of 200-300 nm (Supplementary Note 12). We note that domains with differing spin configurations can affect the unconventional spin torque, since the unconventional spin-Hall conductivity terms can be averaged out to zero under certain symmetry operations (Supplementary Note 11). The fact that we observe non-zero unconventional spin torques in $\mathrm{Mn}_{3} \mathrm{GaN}$, as described below, suggests that certain antiferromagnetic domain configurations are more favorable, which is inferred to be due to a tetragonal distortion that can induce a small non-compensated magnetic moment in $\mathrm{Mn}_{3} \mathrm{GaN}$ thin films. This unbalanced antiferromagnetic domain population is also evidenced by the finite X-ray magnetic linear dichroism (XMLD) signal from the $\mathrm{Mn}_{3} \mathrm{GaN}$ films at the Mn edge for a beam area 100's of microns in scale (Supplementary Note 12).

To measure the symmetry of the spin torques, we use the spintorque ferromagnetic resonance (ST-FMR) technique (Fig. 3a) 15,26. During the ST-FMR measurement, a microwave current applied to $\mathrm{Mn}_{3} \mathrm{GaN}$ produces alternating torques on the Py, and excites the Py magnetic moment into precession, generating a corresponding alternating sinusoidal change of the resistance $R$ due to the anisotropic magnetoresistance (AMR) of Py. We measure a dc voltage signal $V_{\text {mix }}$ across the device bar that arises from the mixing between the alternating current and changes in the device resistance. The resonance in $V_{\text {mix }}$ is obtained by sweeping the external in-plane magnetic field through the Py resonance condition (see "Methods"). Both in-plane and out-of-plane torque components can then be determined individually, as the symmetric and antisymmetric part of the line shape are proportional to the amplitude of the in-plane and out-of-plane torque components, respectively. Considering only the conventional spin-Hall effect (or the Rashba-Edelstein effect and Oersted field), the in-plane and out-ofplane torque components would only have the form of $\mathbf{m} \times(\mathbf{m} \times \mathbf{y})$ and $\mathbf{m} \times \mathbf{y}$, respectively ${ }^{8,27}$. This corresponds to the case of samples containing materials with 2 -fold rotational symmetry, in which case if $\mathbf{m}$ is inverted by rotating the in-plane magnetic field angle $\varphi$ (with
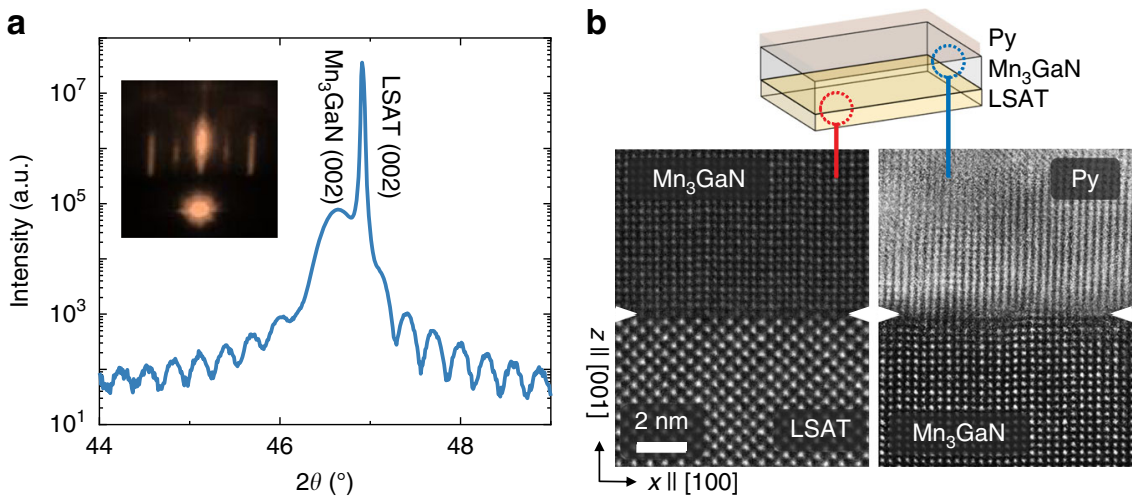

Fig. 2 Structural characterization of the Py/Mn $\mathbf{M}_{\mathbf{3}} \mathbf{G a N} / \mathbf{L S A T}$ a $2 \theta-\omega$ x-ray scan of the heterostructure of $10 \mathrm{~nm} \mathrm{Py} / 30 \mathrm{~nm}$ Mn 3 GaN on LSAT (001) substrate showing single-phase $\mathrm{Mn}_{3} \mathrm{GaN}$ with thickness oscillations indicating a smooth surface and sharp interface with the substrate. Inset shows reflection high-energy electron diffraction (RHEED) pattern of the specular diffraction spot for the $\mathrm{Mn}_{3} \mathrm{GaN}$ surface. $\mathbf{b}$ Scanning transmission electron microscope image of Py/ $\mathrm{Mn}_{3} \mathrm{GaN}$ heterostructure on (001) LSAT substrate with the top Py/Mn $\mathrm{MGaN}_{3}$ interface (left), and the bottom Mn ${ }_{3} \mathrm{GaN} / \mathrm{LSAT}$ interface (right). 
a

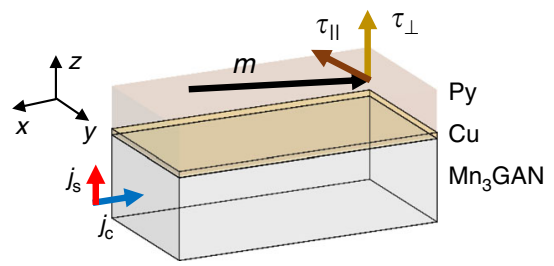

b

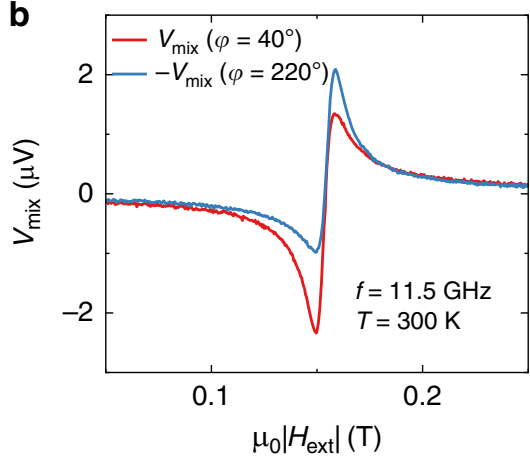

C

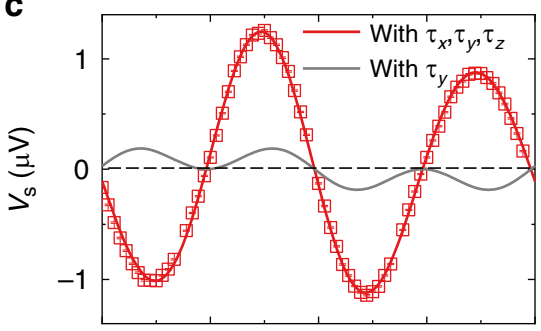

d

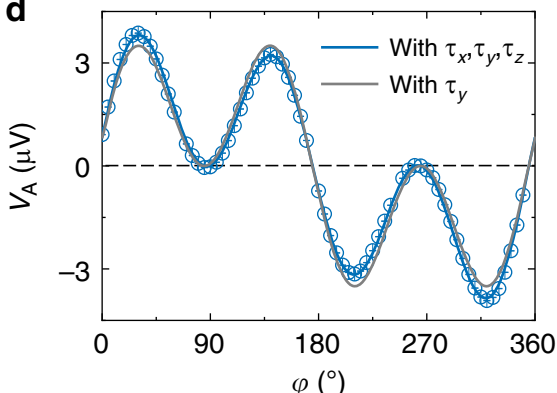

Fig. 3 ST-FMR measurements. a Schematic of the ST-FMR geometry for the Py/Cu/Mn ${ }_{3} \mathrm{GaN}$ structures. $\boldsymbol{\tau}_{\|}$and $\boldsymbol{\tau}_{\perp}$ denote the in-plane and out-of-plane

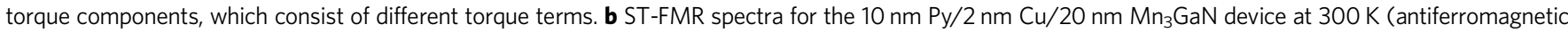
phase) with the Py magnetization oriented at $40^{\circ}$ and $220^{\circ}$ relative to the current axis. c, d Symmetric (c) and antisymmetric (b) ST-FMR components for the $10 \mathrm{~nm} \mathrm{Py} / 2 \mathrm{~nm} \mathrm{Cu} / 20 \mathrm{~nm} \mathrm{Mn}{ }_{3} \mathrm{GaN}$ device as a function of the in-plane magnetic field angle at $300 \mathrm{~K}$. The microwave current is applied along the [100] direction ( $\boldsymbol{x}$ axis). The applied microwave frequency and power are $11.5 \mathrm{GHz}$ and $15 \mathrm{dBm}$, respectively. The error bars indicate fitting uncertainties.

respect to $\mathbf{x}$ ) by $180^{\circ}, V_{\text {mix }}$ must retain the same amplitude but change sign, giving $V_{\text {mix }}(\varphi)=-V_{\text {mix }}\left(\varphi+180^{\circ}\right)$. Any difference in the resonance line shape between $V_{\text {mix }}(\varphi)$ and $-V_{\text {mix }}\left(\varphi+180^{\circ}\right)$ indicates the presence of an additional, unconventional torque component.

Figure $3 \mathrm{~b}$ shows resonance spectra of a $10 \mathrm{~nm} \mathrm{Py/2} \mathrm{nm} \mathrm{Cu/}$ $20 \mathrm{~nm} \mathrm{Mn}{ }_{3} \mathrm{GaN}$ sample with the current flow along the [100] direction for the magnetic field angle $\varphi$ equal to $40^{\circ}$ and $220^{\circ}$, measured at room temperature when the $\mathrm{Mn}_{3} \mathrm{GaN}$ is in the antiferromagnetic state. The $\mathrm{Cu}$ insertion layer breaks the exchange coupling at the $\mathrm{Py} / \mathrm{Mn}_{3} \mathrm{GaN}$ interface, but it allows the transmission of the spin current since $\mathrm{Cu}$ has a long spin diffusion length. We find that the $V_{\text {mix }}\left(40^{\circ}\right)$ and $-V_{\text {mix }}\left(220^{\circ}\right)$ scans are notably different in the antiferromagnetic phase, indicating the presence of unconventional torque components ${ }^{15}$.

To examine the torque components quantitatively, we perform ST-FMR measurements as a function of the in-plane magnetic field angle $\varphi$. Figure $3 c$ and $d$ show the angular dependence of symmetric $V_{\mathrm{S}}$ and antisymmetric $V_{\mathrm{A}}$ part for the $10 \mathrm{~nm} \mathrm{Py} / 2 \mathrm{~nm}$ $\mathrm{Cu} / 20 \mathrm{~nm} \mathrm{Mn}{ }_{3} \mathrm{GaN}$ sample, measured at room temperature. The angular dependence of ST-FMR can be understood as the product of the AMR in $\operatorname{Py}[d R / d \varphi \propto \sin (2 \varphi)]$, with the in-plane $\tau_{\|}$or outof-plane torque $\tau_{\perp}$ components, as $V_{\mathrm{S}} \propto \sin (2 \varphi) \tau_{\|}$and $V_{\mathrm{A}} \propto \sin (2 \varphi) \tau_{\perp}$. For ferromagnetic metal/normal metal bilayers (i.e., $\mathrm{Py} / \mathrm{Pt}$ ), the conventional anti-damping torque $\tau_{\mathrm{y}, \mathrm{AD}} \propto$ $\mathbf{m} \times(\mathbf{m} \times \mathbf{y})$ and field-like torque $\boldsymbol{\tau}_{\mathrm{y}, \mathrm{FL}} \propto \mathbf{m} \times \mathbf{y}$ both have a $\cos (\varphi)$ dependence, giving rise to an overall angular dependence of the form $\sin (2 \varphi) \cos (\varphi)$ for both $V_{\mathrm{S}}$ and $V_{\mathrm{A}}$. We find the angular dependence of both $V_{\mathrm{S}}$ and $V_{\mathrm{A}}$ for the $\mathrm{Mn}_{3} \mathrm{GaN}$ clearly deviate from this simple model (Fig. $3 \mathrm{c}$ and $\mathrm{d}$, gray line), but can be well fitted by adding additional, unconventional torque terms with the presence of spin currents with spin polarizations oriented away from $\mathbf{y}$. The spin currents that are polarized along $\mathbf{x}$ would generate torque $\left[\boldsymbol{\tau}_{\mathrm{x}, \mathrm{AD}} \propto \mathbf{m} \times(\mathbf{m} \times \mathbf{x})\right.$ and $\left.\boldsymbol{\tau}_{\mathrm{x}, \mathrm{FL}} \propto \mathbf{m} \times \mathbf{x}\right]$ with a $\sin (\varphi)$ dependence; while the torques with spin polarization along $\mathbf{z}\left[\boldsymbol{\tau}_{\mathrm{z}, \mathrm{AD}} \propto \mathbf{m} \times(\mathbf{m} \times \mathbf{z})\right.$ and $\left.\boldsymbol{\tau}_{\mathrm{z}, \mathrm{FL}} \propto \mathbf{m} \times \mathbf{z}\right]$, since $\mathbf{m}$ is oriented in the plane, are independent of $\varphi$. We thus fit $V_{\text {mix, }}(\varphi)$ and
$V_{\text {mix, } A}(\varphi)$ to more general forms to take all possible torque terms into account:

$$
\begin{aligned}
& V_{\mathrm{mix}, \mathrm{S}}(\varphi) \propto \sin (2 \varphi)\left(\tau_{\mathrm{x}, \mathrm{AD}} \sin (\varphi)+\tau_{\mathrm{y}, \mathrm{AD}} \cos (\varphi)+\tau_{\mathrm{z}, \mathrm{FL}}\right) \\
& V_{\mathrm{mix}, \mathrm{A}}(\varphi) \propto \sin (2 \varphi)\left(\tau_{\mathrm{x}, \mathrm{FL}} \sin (\varphi)+\tau_{\mathrm{y}, \mathrm{FL}} \cos (\varphi)+\tau_{\mathrm{z}, \mathrm{AD}}\right)
\end{aligned}
$$

We can then find non-zero anti-damping torque terms $\tau_{\mathrm{x}, \mathrm{AD}}$, $\tau_{\mathrm{y}, \mathrm{AD}}$, and $\tau_{\mathrm{z}, \mathrm{AD}}$ (see "Methods" for calculation details) demonstrating the existence of unconventional torque originated from spin polarizations along $\mathbf{x}$ and $\mathbf{z}$. This is consistent with the symmetryallowed spin currents derived from the non-collinear antiferromagnetic $\mathrm{Mn}_{3} \mathrm{GaN}$ magnetic space group through the bulk spin-Hall effect. This mechanism is distinct from those previously reported in noncentrosymmetric systems, and in magnetic trilayers $^{13,14}$. The generation of the spin torque $\tau_{v}, \mathrm{AD}$ relative to the charge current density can be parameterized into the spin-torque ratio $\theta_{\nu}=\frac{\hbar}{2 \mathrm{e}} \frac{j_{s, v}}{j_{c}}$, where $j_{s, v}$ is the spin current density with the spin polarization along $v$ that is absorbed by the $\mathrm{Py}$, and $j_{c}$ is the charge current density in $\mathrm{Mn}_{3} \mathrm{GaN}$ estimated from a parallel-conduction model. We find at room temperature that $\theta_{x}=-0.013 \pm 0.0002$, $\theta_{y}=0.025 \pm 0.0002$ and $\theta_{z}=0.019 \pm 0.0005$, and the spin torque conductivity $\left(\sigma_{z x}^{\nu}=\frac{\theta_{\nu}}{\rho} \frac{\hbar}{2 e}\right.$, where $\rho$ is the charge resistivity of $\left.\mathrm{Mn}_{3} \mathrm{GaN}\right)$ to be around $\sigma_{z x}^{x}=-5.9 \times 10^{3}(\hbar / 2 e)(\Omega m)^{-1}, \sigma_{z x}^{y}=$ $1.1 \times 10^{4}(\hbar / 2 e)(\Omega m)^{-1}$ and $\sigma_{z x}^{z}=8.6 \times 10^{3}(\hbar / 2 e)(\Omega m)^{-1}$ with $\rho=220 \mu \Omega \mathrm{cm}$. The out-of-plane field-like torque has the form $\tau_{\mathrm{y}, \mathrm{FL}}$, dominated by the contribution from the current-induced Oersted field (see "Methods") with no detectable $\tau_{\mathrm{x}, \mathrm{FL}}$ torque. In addition, we observe an in-plane field-like torque $\tau_{z, F L}$ with a large torque ratio of $\theta_{\mathrm{FL}, \mathrm{z}}=-0.15 \pm 0.0002$, which could be generated along with $\tau_{\mathrm{z}, \mathrm{AD}}$ by the spin currents polarized along $\mathbf{z}$. The observed unconventional spin torques could also originate from spin-orbit precession ${ }^{13}$, in which a longitudinal spin polarized current from a ferromagnet can scatter from the ferromagnetic/ normal metal interface. We note that the longitudinal spin polarized current in the non-collinear antiferromagnet and the transverse 
a

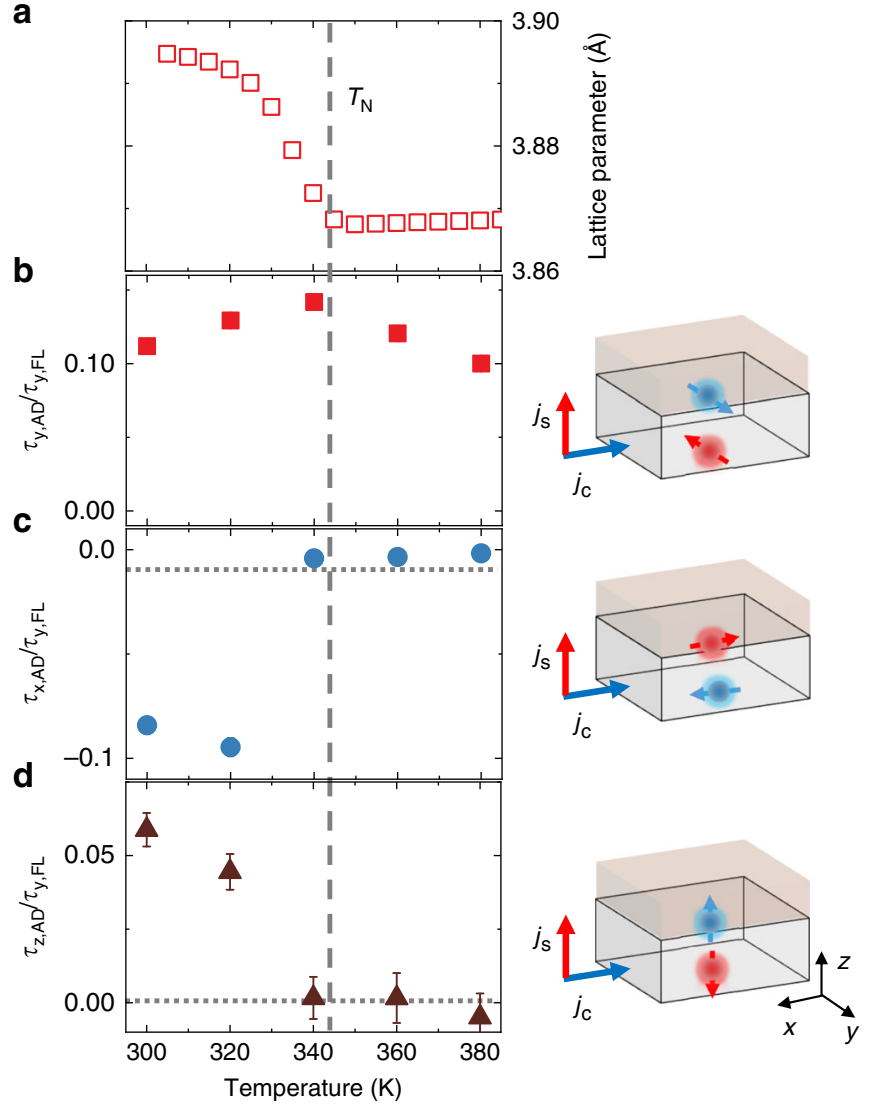

Fig. 4 Temperature dependence of spin-orbit torques. a Out-of-plane

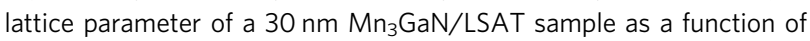
temperature; the lattice parameters anomaly indicates the $\mathrm{Mn}_{3} \mathrm{GaN}$ Néel temperature $T_{\mathrm{N}}$ of $\sim 345 \mathrm{~K}$. b-d The torque ratios $\tau_{\mathrm{y}, \mathrm{AD}} / \tau_{\mathrm{y}, \mathrm{FL}}, \tau_{\mathrm{x}, \mathrm{AD}} / \tau_{\mathrm{y}, \mathrm{FL}}$ and $\tau_{z, \mathrm{AD}} / \tau_{\mathrm{y}, \mathrm{FL}}$ as a function of the temperature for the $10 \mathrm{~nm} \mathrm{Py} / 2 \mathrm{~nm} \mathrm{Cu} /$ $20 \mathrm{~nm} \mathrm{Mn}{ }_{3} \mathrm{GaN}$ device measured at the microwave frequency of $7 \mathrm{GHz}$. The schematics on the right panel show the geometry of the spin-Hall effect with different spin polarizations. The error bars indicate fitting uncertainties. Some of them are smaller than the size of the symbols.

spin current that is reported in this paper could share the same physical origin 28 .

We further confirmed the correlation between the observed unconventional spin polarization and the non-collinear spin structures in $\mathrm{Mn}_{3} \mathrm{GaN}$ by performing angular-dependent STFMR measurements across its antiferromagnetic-to-paramagnetic phase transition. The Néel temperature of the $20 \mathrm{~nm} \mathrm{Mn}_{3} \mathrm{GaN}$ thin film is determined to be $345 \mathrm{~K}$ by tracking the temperature dependence of the out-of-plane lattice parameter (Fig. 4a) because the magnetic phase transition of $\mathrm{Mn}_{3} \mathrm{GaN}$ produces a region of strong negative thermal expansion ${ }^{29}$. Figure $4 \mathrm{~b}-\mathrm{d}$ show the temperature dependence $(300 \mathrm{~K}-380 \mathrm{~K})$ of the ratios between anti-damping torque components and the Oersted torque, $\tau_{\mathrm{y}, \mathrm{AD}} / \tau_{\mathrm{y}, \mathrm{FL}}, \tau_{\mathrm{x}, \mathrm{AD}} / \tau_{\mathrm{y}, \mathrm{FL}}$ and $\tau_{\mathrm{z}, \mathrm{AD}} / \tau_{\mathrm{y}, \mathrm{FL}}$ (extracted from the full angular-dependent ST-FMR measured at each temperature, see Supplementary Note 7). The unconventional torque ratios $\tau_{\mathrm{x}, \mathrm{AD}} / \tau_{\mathrm{y}, \mathrm{FL}}$ and $\tau_{\mathrm{z}, \mathrm{AD}} / \tau_{\mathrm{y}, \mathrm{FL}}$ vanish when the sample temperature is above the Néel temperature, while the conventional component $\tau_{\mathrm{y}, \mathrm{AD}} / \tau_{\mathrm{y}, \mathrm{FL}}$ remains non-zero, with a weak peak near the transition temperature (a similar peak in $\tau_{\mathrm{y}, \mathrm{AD}}$ has been observed near the Curie temperature of $\mathrm{Fe}_{\mathrm{x}} \mathrm{Pt}_{1-\mathrm{x}}$ alloys ${ }^{30}$ ). The vanishing of $\tau_{\mathrm{x}, \mathrm{AD}} / \tau_{\mathrm{y}, \mathrm{FL}}$ and $\tau_{\mathrm{z}, \mathrm{AD}} / \tau_{\mathrm{y}, \mathrm{FL}}$ directly demonstrates the strong correlation between the non-collinear spin structure and the existence of the unconventional spin torques. We also find that the unconventional torques persist at temperatures well below the Néel temperature (Supplementary Note 8), but decrease gradually at lower temperature with the increase of the uncompensated moment and the onset of the anomalous Hall effect in $\mathrm{Mn}_{3} \mathrm{GaN}$ (Supplementary Note 4 and 5). The correlation between the observed unconventional spin-orbit torques, the uncompensated magnetic moment and the anomalous Hall effect in $\mathrm{Mn}_{3} \mathrm{GaN}$ at low temperatures requires further study.

\section{Discussion}

In summary, we have demonstrated the generation of unconventional spin-orbit torque based on low-symmetry non-collinear spin ordering present in the bulk of an epitaxial antiferromagnetic thin film with an antiperovskite structure. Such unconventional torques can be robustly manipulated by controlling the antiferromagnetic ordering across the Néel temperature. This work provides essential insight into understanding how unconventional spin-orbit torques can arise in systems with lower crystalline or magnetic symmetry. This strategy of controlling the spin polarization direction by the design of the spin structure will lead to a much more efficient manipulation and deterministic switching of nano-magnets with arbitrary magnetization, as well as antiferromagnetic tunnel junctions ${ }^{28}$. In addition, our finding offers the possibility to design and control spin currents through manipulating the non-collinear spin order via strain, temperature, chemical doping, and possibly external excitation, opening new areas of research opportunities in antiferromagnetic spintronics ${ }^{31-35}$.

\section{Methods}

Sample growth, fabrication, and characterization. Epitaxial $\mathrm{Mn}_{3} \mathrm{GaN}$ thin films were grown on (001)-oriented LSAT substrates by DC reactive magnetron sputtering using a stoichiometric $\mathrm{Mn}_{3} \mathrm{Ga}$ target in a vacuum chamber with a base pressure of $1 \times 10^{-8}$ Torr. During the growth, the $\mathrm{Mn}_{3} \mathrm{GaN}$ growth mode and surface crystalline structure were monitored by in situ reflection high energy electron diffraction (RHEED). The growth undergoes a $3 \mathrm{D}$ to $2 \mathrm{D}$ growth mode transition. The streaky RHEED pattern after the deposition implies a smooth film surface (Fig. 2a inset). The growth was performed at a substrate temperature of $550{ }^{\circ} \mathrm{C}$ and an $\operatorname{Ar}(62 \mathrm{sccm}) / \mathrm{N}_{2}(8 \mathrm{sccm})$ atmosphere of $10 \mathrm{mTorr}$. After the $\mathrm{Mn}_{3} \mathrm{GaN}$ growth, the sample was cooled down in vacuum. The $\mathrm{Cu}$ and $\mathrm{Py}$ thin films were subsequently sputter deposited at an Ar pressure of 3 mTorr. The atomically flat sample surface was verified using atomic force microscopy (Supplementary Fig. 1). We confirmed the thickness, epitaxial arrangement, and coherence of the $\mathrm{Mn}_{3} \mathrm{GaN}$ films using X-ray reflectivity, X-ray diffraction, and reciprocal space mappings. The growth rate of $\mathrm{Py}$ and $\mathrm{Cu}$ films were calibrated using X-ray reflectivity.

We patterned the samples by using photolithography followed by ion beam milling. Then $200 \mathrm{~nm} \mathrm{Pt} / 5 \mathrm{~nm}$ Ti electrodes were sputter deposited and defined by a lift-off procedure. Devices for ST-FMR were patterned into microstrips (20-50 $\mu \mathrm{m}$ wide and $40-100 \mu \mathrm{m}$ long) with ground-signal-ground electrodes. Devices for electrical transport measurements were patterned into $100 \mu \mathrm{m}$ wide and $500 \mu \mathrm{m}$ long Hall bars.

STEM measurements. The STEM sample was prepared through mechanical polishing down to a thickness of $\sim 10 \mu \mathrm{m}$ by using the precise polishing system (EM TXP, Leica). The polished specimen was then ion-milled using a 1-3 keV Ar ion beam (PIPS II, Gatan) to make the hole for the STEM observation. Afterwards, a low energy milling was performed using $0.1 \mathrm{keV}$ Ar beam to minimize the surface damage from the prior ion-milling process.

The atomic structures were observed using a STEM (JEM-ARM200F, JEOL) at $200 \mathrm{kV}$ equipped with an aberration corrector (ASCOR, CEOS GmbH). The optimum size of the electron probe was $\sim 0.8 \AA$. The collection semi-angles of the HAADF detector were adjusted from 68 to $280 \mathrm{mrad}$ in order to collect large-angle elastic scattering electrons for clear Z-sensitive images. The obtained raw images were processed with a band-pass Wiener filter with a local window to reduce a background noise (HREM research Inc.).

ST-FMR measurements. During ST-FMR measurements, a microwave current at a fixed frequency was applied with the in-plane magnetic field swept from 0 to $0.2 \mathrm{~T}$ for driving the ferromagnetic layer Py through its resonance condition. The amplitude of the microwave current is modulated at a low frequency $(1.713 \mathrm{kHz})$ and the mixing voltage is detected through a lock-in amplifier. For the low temperature ST-FMR measurements (including the room temperature results shown in 
Fig. 3), the device was wire bonded to a coplanar waveguide and then transferred into a liquid helium flow cryostat. For the high temperature measurements (Fig. 4), the sample is placed on a resistive heater with the device probed by the groundsignal-ground rf probe. The ST-FMR resonance line shape can be fitted to a sum of symmetric $V_{\mathrm{S}}$ and antisymmetric $V_{\mathrm{A}}$ Lorentzian components in the form $V_{\text {mix }}=V_{\text {mix }, \mathrm{S}} \frac{W^{2}}{\left(\mu_{0} H_{\text {ext }}-\mu_{0} H_{\mathrm{FMR}}\right)^{2}+W^{2}}+V_{\text {mix }, \mathrm{A}} \frac{W\left(\mu_{0} H_{\text {ext }}-\mu_{0} H_{\mathrm{FMR}}\right)}{\left(\mu_{0} H_{\mathrm{ext}}-\mu_{0} H_{\mathrm{FMR}}\right)^{2}+W^{2}}$, where $W$ is the halfwidth-at-half-maximum resonance linewidth, $\mu_{0}$ is the permeability in vacuum and $H_{\mathrm{FMR}}$ is the resonance field. The in-plane $\tau_{\|}$and out-of-plane $\tau_{\perp}$ components are proportional to $V_{\text {mix,S }}$ and $V_{\text {mix,A }}$ components, which can be expressed as,

$$
\begin{gathered}
V_{\mathrm{mix}, \mathrm{S}}=-\frac{I_{\mathrm{rf}}}{2}\left(\frac{d R}{d \varphi}\right) \frac{1}{\alpha\left(2 \mu_{0} H_{\mathrm{FMR}}+\mu_{0} M_{\mathrm{eff}}\right)} \tau_{\|} \\
V_{\mathrm{mix}, \mathrm{A}}=-\frac{I_{\mathrm{rf}}}{2}\left(\frac{d R}{d \varphi}\right) \frac{\sqrt{1+M_{\mathrm{eff}} / H_{\mathrm{FMR}}}}{\alpha\left(2 \mu_{0} H_{\mathrm{FMR}}+\mu_{0} M_{\mathrm{eff}}\right)} \tau_{\perp}
\end{gathered}
$$

where $I_{\mathrm{rf}}$ is the microwave current, $R$ is the device resistance as a function of inplane magnetic field angle $\varphi$ due to the AMR of Py, $\alpha$ is the Gilbert damping coefficient, and $M_{\text {eff }}$ is the effective magnetization. The AMR of Py is determined by measuring the device resistance as a function of magnetic field angle with a field magnitude of $0.1 \mathrm{~T}$. We calibrate the microwave current $I_{\mathrm{rf}}$ by measuring the microwave current-induced device resistance change due to Joule heating ${ }^{36,37}$ (Supplementary Note 6). The in-plane and out-of-plane torques can be expressed as the angular dependence of the torque components with different spin polarization directions,

$$
\begin{aligned}
& \tau_{\|}=\tau_{\mathrm{x}, \mathrm{AD}} \sin (\varphi)+\tau_{\mathrm{y}, \mathrm{AD}} \cos (\varphi)+\tau_{\mathrm{z}, \mathrm{FL}} \\
& \tau_{\perp}=\tau_{\mathrm{x}, \mathrm{FL}} \sin (\varphi)+\tau_{\mathrm{y}, \mathrm{FL}} \cos (\varphi)+\tau_{\mathrm{z}, \mathrm{AD}}
\end{aligned}
$$

The strength of the torque components can then be determined from Eqs. (3-6) with the calibrated $I_{\mathrm{rf}}$ values, from which we noticed that the primary contribution to $\tau_{\mathrm{y}, \mathrm{FL}}$ is the current-induced Oersted field. The spin torque ratios can be expressed as,

$$
\begin{aligned}
& \theta_{x}=\frac{\tau_{\mathrm{x}, \mathrm{AD}}}{\tau_{\mathrm{y}, \mathrm{FL}}} \frac{\mathrm{e} \mu_{0} M_{\mathrm{s}} t_{\mathrm{Py}} t_{\mathrm{MGN}}}{\hbar} \\
& \theta_{y}=\frac{\tau_{\mathrm{y}, \mathrm{AD}}}{\tau_{\mathrm{y}, \mathrm{FL}}} \frac{\mathrm{e} \mu_{0} M_{\mathrm{s}} t_{\mathrm{Py}} t_{\mathrm{MGN}}}{\hbar} \\
& \theta_{z}=\frac{\tau_{\mathrm{z}, \mathrm{AD}}}{\tau_{\mathrm{y}, \mathrm{FL}}} \frac{\mathrm{e} \mu_{0} M_{\mathrm{s}} t_{\mathrm{Py}} t_{\mathrm{MGN}}}{\hbar}
\end{aligned}
$$

where $M_{\mathrm{s}}$ and $t_{\mathrm{Py}}$ are the saturation magnetization and the thickness of Py; $t_{\mathrm{MGN}}$ is the thickness of $\mathrm{Mn}_{3} \mathrm{GaN}$. $\hbar$ is the reduced Planck's constant and e is the electron charge. The saturation magnetization of Py was measured with SQUID magnetometry, and is indistinguishable from the effective magnetization determined by ST-FMR.

Electrical transport measurements of $\mathbf{M n}_{3} \mathbf{G a N}$. Electrical transport measurements of $\mathrm{Mn}_{3} \mathrm{GaN}$ films were performed directly on as-grown films wire-bonded in a four-corner van der Pauw geometry. Both sheet resistance and Hall resistance were measured as a function of temperature and magnetic induction in a Quantum Design Physical Property Measurement System. Film resistivity was computed by solving the van der Pauw equation in conjunction with film thickness as measured with $\mathrm{x}$-ray reflectivity, while Hall resistance was calculated by summing two orthogonal Hall configurations. The longitudinal resistivity and the Hall resistance of $\mathrm{Mn}_{3} \mathrm{GaN}$ films vs. temperature are reported in Supplementary Fig. 4.

Temperature dependence of neutron diffraction. Single crystal neutron diffraction measurements were performed on the WISH time-of-flight diffractometer ${ }^{38}$ at ISIS, the UK neutron and muon source. A stack of eight, approximately $250 \mathrm{~nm}$ thick (001) $\mathrm{Mn}_{3} \mathrm{GaN}$ film samples with lateral dimensions $10 \times 8 \mathrm{~mm}$, were co-aligned and oriented for the measurement of nuclear and magnetic diffraction intensities in the (HK0) reciprocal lattice plane. The sample was first mounted within a ${ }^{4} \mathrm{He}$ cryostat, and diffraction patterns were collected from a base temperature of $1.5 \mathrm{~K}$ up to $300 \mathrm{~K}$, in $25 \mathrm{~K}$ steps. The sample was transferred to a medium-range furnace, and diffraction patterns were then collected at $320,340,360$, and $390 \mathrm{~K}$.

Temperature dependence of X-ray diffraction. The X-ray diffraction data were acquired at beamline 6-ID-B at the Advanced Photon Source with $12 \mathrm{keV}$ incident $\mathrm{X}$-ray energy. The sample temperature was controlled employing an ARS high temperature cryostat. Data were collected with $5 \mathrm{~K}$ steps; and at each temperature the sample position was realigned with respect to the base-temperature reciprocal space matrix. The sample was mounted on a standard PSI Huber diffractometer. The representative temperature dependence of $\mathrm{x}$-ray diffraction spectra around the LSAT (003) reflection can be found in Supplementary Fig. 2.
Theoretical calculations. The electronic band structure of $\mathrm{Mn}_{3} \mathrm{GaN}$ was calculated by using first-principles density functional theory (DFT) with Quantum ESPRESSO ${ }^{39}$ and fully relativistic ultrasoft pseudopotentials ${ }^{40}$. The exchange and correlation effects were treated within the generalized gradient approximation $(\mathrm{GGA})^{41}$. The plane-wave cut-off energy of $57 \mathrm{Ry}$ and a $16 \times 16 \times 16 k$-point mesh in the irreducible Brillouin zone were used in the calculations. Spin-orbit coupling and non-collinear $\Gamma^{5 \mathrm{~g}}$ antiferromagnetism were included in all electronic structure calculations. We note that even though the spin-orbit coupling in $\mathrm{Mn}_{3} \mathrm{GaN}$ is relatively small, it still plays an important role to couple the spin and the lattice, which lifts the spin rotation symmetry and allows the existence of the non-vanishing spin-Hall conductivity (Supplementary Note 10). The calculated band structures for $\mathrm{Mn}_{3} \mathrm{GaN}$ in antiferromagnetic and paramagnetic phases are shown in Supplementary Fig. 9.

The spin-Hall effect is given by ${ }^{42}$

$$
\sigma_{i j}^{k}=\frac{e^{2}}{\hbar} \int \frac{d^{3} \vec{k}}{(2 \pi)^{3}} \sum_{n} f_{n \vec{k}} \Omega_{n, i j}^{k}(\vec{k})
$$

$$
\Omega_{n, i j}^{k}(\vec{k})=-2 \operatorname{Im} \sum_{n \neq n^{\prime}} \frac{\left\langle n \vec{k}\left|J_{i}^{k}\right| n^{\prime} \vec{k}\right\rangle\left\langle n^{\prime} \vec{k}\left|v_{j}\right| n^{\prime} \vec{k}\right\rangle}{\left(E_{n \vec{k}}-E_{n^{\prime} \vec{k}}\right)^{2}}
$$

where $f_{n \vec{k}}$ is the Fermi-Dirac distribution for the $n$th band, $J_{i}^{k}=\frac{1}{2}\left\{v_{i}, s_{k}\right\}$ is the spin current operator with spin operator $S_{k}, v_{j}=\frac{1}{\hbar} \frac{\partial H}{\partial k_{j}}$ is the velocity operator, and $i, j, k=x, y, z . \Omega_{n, i j}^{k}(\vec{k})$ is referred to as the spin Berry curvature in analogy to the ordinary Berry curvature. In order to calculate the spin- Hall conductivities, we construct the tight-binding Hamiltonians using PAOFLOW code $^{43}$ based on the projection of the pseudo-atomic orbitals (PAO) ${ }^{44,45}$ from the non-self-consistent calculations with a $16 \times 16 \times 16 k$-point mesh. The spin-Hall conductivities were calculated using the tight-binding Hamiltonians with a $48 \times 48 \times 48 k$-point mesh by the adaptive broadening method to get the converged values.

Synchrotron spectroscopy and microscopy. X-ray magnetic circular dichroism (XMCD) and X-ray magnetic linear dichroism (XMLD) spectroscopy were measured at beamline 4.0.2, and X-ray microscopy at beamline 11.0.1.1 (PEEM-3) at the Advanced Light Source (ALS). In spectroscopy, total-electronyield mode was employed by monitoring the sample drain current, and a grazing incidence angle of $30^{\circ}$ to the sample surface along the [110] direction to probe the magnetic state. The obtained dichroism energies giving information on the magnetic nature of the $\mathrm{Mn}_{3} \mathrm{GaN}$ were then used to image the domain texture by X-ray photoemission electron microscopy (XPEEM), also taken with Xrays at a $30^{\circ}$ grazing incidence along the [110] direction. Images taken at the maximum dichroism energies as a function of polarization were normalized by pre-edge energy images in order to minimize any topographic and work function contrast while emphasizing the magnetic contrast of the $\mathrm{Mn}_{3} \mathrm{GaN}$ films.

\section{Data availability}

The data that support the findings of this study are available from the corresponding author on reasonable request.

Received: 15 February 2020; Accepted: 22 July 2020; Published online: 16 September 2020

\section{References}

1. Ando, K. et al. Electric manipulation of spin relaxation using the spin Hall effect. Phys. Rev. Lett. 101, 036601 (2008).

2. Gambardella, P. \& Miron, I. M. Current-induced spin-orbit torques. Philos. Trans. R. Soc. A Math. Phys. Eng. Sci. 369, 3175-3197 (2011).

3. Brataas, A., Kent, A. D. \& Ohno, H. Current-induced torques in magnetic materials. Nat. Mater. 11, 372-381 (2012).

4. Liu, L. et al. Spin-torque switching with the giant spin Hall effect of tantalum. Science 336, 555-558 (2012)

5. Kim, J. et al. Layer thickness dependence of the current-induced effective field vector in $\mathrm{Ta}|\mathrm{CoFeB}| \mathrm{MgO}$. Nat. Mater. 12, 240-245 (2013).

6. Emori, S., Bauer, U., Ahn, S.-M., Martinez, E. \& Beach, G. S. D. Currentdriven dynamics of chiral ferromagnetic domain walls. Nat. Mater. 12, 611-616 (2013).

7. Fan, X. et al. Quantifying interface and bulk contributions to spin-orbit torque in magnetic bilayers. Nat. Commun. 5, 3042 (2014).

8. Mellnik, A. R. et al. Spin-transfer torque generated by a topological insulator. Nature 511, 449-451 (2014).

9. Dyakonov, M. I. \& Perel, V. I. Current-induced spin orientation of electrons in semiconductors. Phys. Lett. A 35, 459-460 (1971).

10. Sinova, J., Valenzuela, S. O., Wunderlich, J., Back, C. H. \& Jungwirth, T. Spin Hall effects. Rev. Mod. Phys. 87, 1213-1260 (2015). 
11. Fukami, S., Anekawa, T., Zhang, C. \& Ohno, H. A spin-orbit torque switching scheme with collinear magnetic easy axis and current configuration. Nat. Nanotechnol. 11, 621-625 (2016).

12. Amin, V. P. \& Stiles, M. D. Spin transport at interfaces with spin-orbit coupling: phenomenology. Phys. Rev. B 94, 104420 (2016).

13. Baek, S. H. C. et al. Spin currents and spin-orbit torques in ferromagnetic trilayers. Nat. Mater. 17, 509-513 (2018).

14. Humphries, A. M. et al. Observation of spin-orbit effects with spin rotation symmetry. Nat. Commun. 8, 911 (2017).

15. MacNeill, D. et al. Control of spin-orbit torques through crystal symmetry in $\mathrm{WTe}_{2} /$ ferromagnet bilayers. Nat. Phys. 13, 300-305 (2016).

16. Fang, D. et al. Spin-orbit-driven ferromagnetic resonance. Nat. Nanotechnol. 6, 413-417 (2011).

17. Kurebayashi, H. et al. An antidamping spin-orbit torque originating from the Berry curvature. Nat. Nanotechnol. 9, 211-217 (2014).

18. Ciccarelli, C. et al. Room-temperature spin-orbit torque in NiMnSb. Nat. Phys. 12, 855-860 (2016).

19. Zhang, W. et al. Spin Hall effects in metallic antiferromagnets. Phys. Rev. Lett. 113, 196602 (2014)

20. Zhang, W. et al. Giant facet-dependent spin-orbit torque and spin Hall conductivity in the triangular antiferromagnet $\mathrm{IrMn}_{3}$. Sci. Adv. 2, e1600759 (2016).

21. Fukami, S., Zhang, C., DuttaGupta, S., Kurenkov, A. \& Ohno, H. Magnetization switching by spin-orbit torque in an antiferromagnet-ferromagnet bilayer system. Nat. Mater. 15, 535-541 (2016).

22. Kimata, M. et al. Magnetic and magnetic inverse spin Hall effects in a noncollinear antiferromagnet. Nature 565, 627-630 (2019)

23. Bertaut, E. F., Fruchart, D., Bouchaud, J. P. \& Fruchart, R. Diffraction neutronique de $\mathrm{Mn}_{3} \mathrm{GaN}$. Solid State Commun. 6, 251-256 (1968).

24. Shi, $\mathrm{K}$. et al. Baromagnetic effect in antiperovskite $\mathrm{Mn}_{3} \mathrm{Ga}_{0.95} \mathrm{~N}_{0.94}$ by neutron powder diffraction analysis. Adv. Mater. 28, 3761-3767 (2016).

25. Matsunami, D., Fujita, A., Takenaka, K. \& Kano, M. Giant barocaloric effect enhanced by the frustration of the antiferromagnetic phase in Mn3GaN. Nat. Mater. 14, 73-78 (2015).

26. Liu, L., Moriyama, T., Ralph, D. C. \& Buhrman, R. A. Spin-torque ferromagnetic resonance induced by the spin Hall effect. Phys. Rev. Lett. 106, 036601 (2011)

27. Garello, K. et al. Symmetry and magnitude of spin-orbit torques in ferromagnetic heterostructures. Nat. Nanotechnol. 8, 587-593 (2013)

28. Železný, J., Zhang, Y., Felser, C. \& Yan, B. Spin-polarized current in noncollinear antiferromagnets. Phys. Rev. Lett. 119, 187204 (2017).

29. Takenaka, K. \& Takagi, H. Giant negative thermal expansion in Ge-doped anti-perovskite manganese nitrides. Appl. Phys. Lett. 87, 261902 (2005).

30. Ou, Y., Ralph, D. C. \& Buhrman, R. A. Strong enhancement of the spin Hall effect by spin fluctuations near the Curie point of $\mathrm{FexPt}_{1-\mathrm{x}}$ alloys. Phys. Rev. Lett. 120, 097203 (2018).

31. MacDonald, A. H. \& Tsoi, M. Antiferromagnetic metal spintronics. Philos. Trans. R. Soc. A Math. Phys. Eng. Sci. 369, 3098-3114 (2011).

32. Jungwirth, T., Marti, X., Wadley, P. \& Wunderlich, J. Antiferromagnetic spintronics. Nat. Nanotechnol. 11, 231-241 (2016).

33. Wadley, P. et al. Electrical switching of an antiferromagnet. Science 351, 587-590 (2016).

34. Železný, J., Wadley, P., Olejník, K., Hoffmann, A. \& Ohno, H. Spin transport and spin torque in antiferromagnetic devices. Nat. Phys. 14, 220-228 (2018).

35. Baltz, V. et al. Antiferromagnetic spintronics. Rev. Mod. Phys. 90, 015005 (2018).

36. Tshitoyan, V. et al. Electrical manipulation of ferromagnetic NiFe by antiferromagnetic IrMn. Phys. Rev. B 92, 214406 (2015).

37. Zhang, W. et al. All-electrical manipulation of magnetization dynamics in a ferromagnet by antiferromagnets with anisotropic spin Hall effects. Phys. Rev. B 92, 144405 (2015).

38. Chapon, L. C. et al. Wish: The new powder and single crystal magnetic diffractometer on the second target station. Neutron N. 22, 22-25 (2011).

39. Giannozzi, P. et al. QUANTUM ESPRESSO: A modular and open-source software project for quantum simulations of materials. J. Phys. Condens. Matter 21, 395502 (2009).

40. Vanderbilt, D. Soft self-consistent pseudopotentials in a generalized eigenvalue formalism. Phys. Rev. B 41, 7892-7895 (1990).

41. Perdew, J. P., Burke, K. \& Ernzerhof, M. Generalized gradient approximation made simple. Phys. Rev. Lett. 77, 3865-3868 (1996).

42. Gradhand, M. et al. First-principle calculations of the Berry curvature of Bloch states for charge and spin transport of electrons. J. Phys. Condens. Matter 24, 213202 (2012)

43. Buongiorno Nardelli, M. et al. PAOFLOW: A utility to construct and operate on ab initio Hamiltonians from the projections of electronic wavefunctions on atomic orbital bases, including characterization of topological materials. Comput. Mater. Sci. 143, 462-472 (2018).
44. Agapito, L. A., Ferretti, A., Calzolari, A., Curtarolo, S. \& Buongiorno Nardelli, M. Effective and accurate representation of extended Bloch states on finite Hilbert spaces. Phys. Rev. B 88, 165127 (2013).

45. Agapito, L. A., Ismail-Beigi, S., Curtarolo, S., Fornari, M. \& Nardelli, M. B. Accurate tight-binding Hamiltonian matrices from ab initio calculations: Minimal basis sets. Phys. Rev. B 93, 035104 (2016).

\section{Acknowledgements}

This work was supported by the National Science Foundation (NSF) under DMREF Grant DMR-1629270, the University of Wisconsin Materials Research Science and Engineering Center (DMR-1720415), the Army Research Office through Grant W911NF-17-1-0462 and AFOSR FA9550-15-1-0334. Transport and magnetization measurement at the University of Wisconsin-Madison was supported by the US Department of Energy (DOE), Office of Science, Office of Basic Energy Sciences under Award DE-FG02-06ER46327. Theoretical research at University of Nebraska-Lincoln (UNL) was partly supported by the NSF Materials Research Science and Engineering Center (MRSEC) program under Grant DMR-1420645. Computations were performed at the UNL's Holland Computing Center. Work at Cornell was supported by the NSF (DMR-1708499), and was performed in part at the Cornell NanoScale Facility, an NNCI member supported by NSF Grant NNCI-1542081 and also in part in the shared facilities of the Cornell Center for Materials Research which are supported by the NSF MRSEC program (DMR-1719875). This research used resources of the Advanced Photon Source, a U.S. DOE Office of Science User Facility operated for the DOE Office of Science by Argonne National Laboratory under Contract No. DE-AC02-06CH11357. This research used resources of the Advanced Light Source, which is a DOE Office of Science User Facility under contract no. DE-AC02-05CH11231.

\section{Author contributions}

T.N. and C.B.E. conceived the research. C.B.E., M.S.R., E.Y.T., D.C.R, P.G.R., T.T., and S.Y.C. supervised the experiments. T.N., C.X.Q., and L.G. performed the sample growth and surface/structural characterizations. T.N. performed the device fabrication. T.N. and J.G performed ST-FMR measurements and analysis. N.C. and J.I. performed magnetic and electrical transport characterizations. G.G. and D.F.S. performed the theoretical calculations. K.S. and S.Y.C. carried out the high-resolution TEM experiments. R.D.J., P.M., and P.G.R. conceived and performed the neutron diffraction experiment. P.R., J.W.K., and Y.S.C. carried out the synchrotron diffraction measurements. R.V.C. and I.H performed synchrotron spectroscopy measurements, and R.V.C. performed and analyzed synchrotron microscopy measurements. T.N., J.I., D.F.S., D.C.R., and C.B.E. wrote the paper. All authors discussed the results and commented on the paper. C.B.E. directed the research.

\section{Competing interests}

T.N. and C.B.E. have filed a patent on the technology in this paper. The remaining authors declare no competing interests.

\section{Additional information}

Supplementary information is available for this paper at https://doi.org/10.1038/s41467 020-17999-4.

Correspondence and requests for materials should be addressed to C.B.E.

Peer review information Nature Communications thanks the anonymous reviewer(s) for their contribution to the peer review of this work.

Reprints and permission information is available at http://www.nature.com/reprints

Publisher's note Springer Nature remains neutral with regard to jurisdictional claims in published maps and institutional affiliations.

pen Access This article is licensed under a Creative Commons Attribution 4.0 International License, which permits use, sharing, adaptation, distribution and reproduction in any medium or format, as long as you give appropriate credit to the original author(s) and the source, provide a link to the Creative Commons license, and indicate if changes were made. The images or other third party material in this article are included in the article's Creative Commons license, unless indicated otherwise in a credit line to the material. If material is not included in the article's Creative Commons license and your intended use is not permitted by statutory regulation or exceeds the permitted use, you will need to obtain permission directly from the copyright holder. To view a copy of this license, visit http://creativecommons.org/ licenses/by/4.0/.

(C) The Author(s) 2020 